\title{
INTRODUCTION
}

Optical spectroscopy is a long-established technique for the observation of oxygenation and haemodynamic effects in tissue, although the specific popularity of near-infrared spectroscopy (NIRS) largely followed the 1977 publication of Jöbsis, which showed its possible use as a non-invasive monitor of cerebral oxygenation. Since that time, many hundreds of papers have been published demonstrating its potential not only in the monitoring of the brain (Benaron, Chance, Cooper, Gratton, Kirkpatrick, Levy, Tamura, Villringer and Wyatt papers in this volume) but also in muscle (Chance, Ferrari), the breast (Tromberg), liver and kidney (Thorniley). Although many of these studies have been in animal models, increasingly the technique is being used in clinical research, most widely in the neonatal area (Benaron, Wyatt), but also now in adult patients (Chance, Kirkpatrick, Levy). Almost all the studies reported to date have used what one might call 'conventional' NIRS technology which just measures changes in optical density at several wavelengths. However, in the past few years, new instruments involving either time- or frequency-resolved measurements have been developed which may allow the accurate quantitative measurement of tissue optical properties (Chance, Delpy, Gratton, Tamura, Tromberg). In the last ten years, there has also been an increasing focus on the possibilities of imaging with NIR light, and hence monitoring regional changes in oxygenation and haemodynamics (Arridge, Benaron, Chance, Delpy, Gratton). The meeting on 'Near-Infrared Spectroscopy and Imaging of Living Systems', held at the Royal Society, was intended to bring together the basic scientists, engineers and clinicians working in this area to try and review the existing abilities of the technique, summarize the outstanding problems, and identify the possible future directions for research in this area. The papers in this volume summarize the presentations made at the meeting by some of the leading research groups in the world. However, the meeting was also attended by a large audience who contributed considerably to the discussion that followed both each paper and each session, and this introduction aims to try and highlight some of the points raised in those discussions. In order to provide some structure to this, these points have been organized under general topic headings.

\section{(a) Haemodynamics}

'Conventional' NIRS has extremely high temporal resolution compared to most non-invasive methods, and this has enabled the observation of rapid dynamic changes in tissue haemodynamics. One topic of considerable recent interest has been its potential for monitoring the haemodynamic changes associated with neuronal activation (Chance, Tamura, Villringer), although newer technology may be required to monitor the small scattering changes associated with the initial membrane depolarization event (Gratton). Simple optical density changes alone, measured on the surface of the head, have been shown to enable the detection of haematomas (Chance, Levy). However, the exact mechanism of this was contested since modelling of the light distribution in the head seems to indicate relatively little penetration into central white matter (Delpy, Arridge).

\section{(b) Other measurements}

There was some debate as to which NIRS parameters were related to tissue damage/energy failure. The above discussion concluded that changes in haemoglobin oxygenation (so-called 'trend monitoring') have been shown to correlate with possible damage to tissues. However, in principle, there are more powerful signals that can be used in this respect. The initial hope in this area was the cytochrome oxidase signal. There was general agreement that this signal could, in principle, be an accurate monitor of large drops in intracellular oxygen partial pressure. Recent studies (Cooper) suggest that it could also be related to tissue energy failure, but this was contested in the discussion. The availability of new instrumentation (time, phase or full spectrally resolved measurements) mean that in addition to the existing NIRS parameters, the true optical path length and tissue scattering coefficient can now be measured. A case was put (Chance) that scattering changes will be a very useful measure of membrane depolarization, and therefore of energy failure in both animals and clinical subjects. Although some groups questioned the size of the scattering changes observed, this area is likely to be of great interest in years to come.

One of the most interesting areas of development is the combination of molecular biology and photon counting developed by the Benaron group. Although using this technology in humans may be a long way off (at least until gene therapy becomes routine), in animal models it provides a potentially limitless tool. The ability to tag genes with NIR/optical probes could provide the ability to monitor gene activation in vivo. Thus, in the future in vivo molecular biology may be as easy to do as in vivo haemodynamic and bioenergetic monitoring are now. 


\section{(c) Imaging}

Unlike NIRS, NIR imaging (NIRI) has yet to mature as a field with few images being produced from in vivo measured data. Tissue optical properties (essential for the modelling involved in the reconstruction of an image) are becoming better understood (Delpy, Gratton, Tromberg), and more sophisticated reconstruction algorithms (Arridge) are now coming on-line; it was stressed in the discussion that even without these, interesting data could often be obtained even with simple algorithms (e.g. back projection). As with spectroscopy this is an area where the smaller size, easier geometry, and more amenable optical properties of the infant or baby brain is likely to yield the first breakthroughs (Arridge, Benaron, Delpy). An area not discussed in these papers in detail, but one on which there was much discussion was breast imaging for the early detection of tumours (Gratton, Tromberg). It is here that commercial companies have put the most resources with at least two systems now undergoing clinical testing.

\section{(d) Functional imaging}

Of great recent interest has been the ability of NIRS to detect functional activation in the adult brain (Chance, Gratton, Tamura, Villringer). There was some debate about the size of the haemodynamic changes observed, with some authors (Chance) seeing much larger changes than others (Villringer). This may be resolved as different groups harmonize their measurement techniques (e.g. optode localization) and as we increase our understanding of the differences in response to the varying instrumentation used (Delpy). Although the resolution achievable with NIR will never be at the level of fMRI (functional magnetic resonance imaging), the general advantages that NIR has over MRI in expense, portability, temporal resolution and ease of use were exemplified in the large-scale study of functional activation described by Chance in schoolchildren, a project that would have been vastly more difficult to undertake with MRI techniques.

\section{(e) Where the signal comes from}

There was an extensive discussion over which region of tissue NIR instruments interrogate. This is especially important in studies on the adult head, where there is a well-known significant contribution from the extracerebral tissues (skin, skull, cerebrospinal fluid (CSF)). This causes problems in interpreting unexpected changes in oxygenation and blood volume as measured by NIRS (Kirkpatrick). This debate should not overshadow the fact that some light clearly does report on cerebral events, as witnessed by the haematoma detection and functional imaging studies that have been reported.

\section{(f) The future}

NIRS has a clear future as the easiest non-invasive monitor of tissue haemoglobin oxygenation and concentration changes. The technology (especially GCD detection systems) is such, that even in the absence of a commercial instrument manufacturer it is relatively easy to custom-build machines for sensitive detection of any required wavelength of light in the NIR. This area of research is likely to continue as long as there are physiological and pathophysiological questions to be answered in this area.

New technology, however, clearly holds the key if NIR is to break into the clinical arena. The major technical advances that the discussants thought most useful would be reliable absolute haemoglobin concentration measurements (without the need for any physiological manipulation) and high resolution images. There still remains the question that if all these goals are achieved, what will be its clinical value? We feel it is likely that NIR will find its major initial clinical use either in an area where there is a clear clinical requirement for large-scale diagnostics (e.g. breast imaging) or in clinical environments where the use of high technology is already well-established and the patient is easy to monitor (e.g. intensive care or surgery).

March 1997

C. Cooper

D. T. Delpy 